\title{
PERSPECTIVA DE LA DINÁMICA DE DESARROLLO ECONÓMICO Y CATEGORÍAS DE VALOR DEL SUELO URBANO EN EL SECTOR EL PRADO DE LA CIUDAD DE BARRANQUILLA, COLOMBIA'
}

\author{
Artículo de Reflexión - Recibido: 20 de Marzo de 2016 - Aceptado: 30 de Mayo de 2016
}

Humberto Manuel Osorio Chávez²

Universidad de la Costa CUC. Barranquilla, Colombia. hosorio1@cuc.edu.co

Para citar este artículo / to reference this article:

Osorio, H. (2016). Perspectiva de la dinámica de desarrollo económico y categorías de valor del suelo urbano en el sector el prado de la ciudad de Barranquilla, Colombia. Módulo Arquitectura CUC, Vol.17 №1, 63-74.

\begin{abstract}
Resumen
El artículo plantea una aproximación en las categorías de valor de suelo urbano existentes en El Prado del Distrito de Barranquilla, de manera correlacionada con los precios de suelo que actualmente se manejan en el sector, los cuales, permitieron construir una línea base de indicadores urbanos de usos y valor del suelo categorizados. Esta información se articuló con la visión y acciones de desarrollo del sector y la ciudad de Barranquilla desde las bases del desarrollo urbano y territorial. Este ejercicio investigativo desarrolló un análisis predio a predio, de las tipologías de viviendas y los valores de suelos actuales, partiendo de una metodología de trabajo de campo para el tratamiento de datos geo-referenciados y sistematizados. Se buscó identificar la coherencia que existe entre los tipos de edificaciones y el valor referencial, desde las bases normativas que rigen esa dinámica urbana e inmobiliaria en la ciudad y el país.
\end{abstract}

\section{Palabras clave}

Análisis predial, Valor del suelo, Desarrollo urbano, Perspectiva económica, tipología edificatoria.

\footnotetext{
1 Artículo elaborado con base en el proyecto "Tipología de vivienda y valores en el suelo en el distrito de Barranquilla, sector El Prado". Grupo ARUCO adscrito a la Facultad de Arquitectura de la Universidad de la Costa CUC, en el marco de la línea de investigación Calidad del Hábitat y el Entorno. Autor: Humberto Manuel Osorio Chávez.

2 Arquitecto; Especialista en Estudios Pedagógicos, y en Gerencia de Proyectos de Construcciones. Candidato a Magíster en Urbanismo y Desarrollo Territorial. Docente Tiempo Completo adscrito a la Facultad de Arquitectura de la Universidad de la Costa CUC.
} 


\title{
PERSPECTIVE OF THE DYNAMICS OF ECONOMIC DEVELOPMENT AND CATEGORIES OF VALUE OF URBAN LAND IN THE SECTOR "EL PRADO" CITY OF BARRANQUILLA, COLOMBIA.
}

\begin{abstract}
The article raises an approach in the categories of value of existing urban land in El Prado Barranquilla District, correlated way with the prices of land is now working in the sector, which allowed to build a base line of urban indicators uses and value categorized ground. This information was articulated with the vision and actions of development of the sector and the city of Barranquilla from the bases of urban and regional development. This investigative exercise developed an estate property analysis of the types of housing and current land values, starting from a fieldwork methodology for the treatment of geo -referenced data and systematized. We sought to identify the consistency between the types of buildings and the reference value from the normative basis governing the urban and real estate dynamics in the city and country.
\end{abstract}

Keywords

Analysis of land, Land value, Urban Development, Economic Development, Building types. 


\section{Introducción}

Entre los años 1916 y 1922 se presentaron dos hechos de suma importancia en la configuración y el futuro urbano de la ciudad de Barranquilla. En 1916 se generó la primera invasión urbana conformada por 6 manzanas del barrio Montecristo, con pobladores desplazados de la ribera del rio Magdalena y en 1922 se construyó la primera urbanización El Prado, primera en el país. De aquí en adelante, estos dos extremos, caracterizarían el desarrollo urbano de la ciudad. Pero también, aunque en menor incidencia que las invasiones irregulares de tierras, la falta de políticas de recuperación de plusvalías y la desigualdad en el cobro de impuestos territoriales en las ciudades latinoamericanas influye fuertemente y de manera negativa en el desarrollo urbano. (Alfonso y Erba, 2015)

La urbanización El Prado represento el 55\% del desarrollo urbano en expansión de la época en Barranquilla y se ubicaba al lado del barrio Las Quintas y limitando con la carrera 50 antiguo camino a la playa. El terreno es el más alto de toda esa planicie y se lograba tener una excelente vista del rio Magdalena y hasta la Sierra Nevada de Santa Marta. Desde su inicio fue pensada como una urbanización que satisficiera las nuevas exigencias o necesidades de comerciantes acaudalados y familias de la elite local. (Ospino, 2003)

En Colombia se levantaron múltiples censos de población durante toda su historia como en la época de la colonia fueron cinco en los años 1770, 1778, 1782, 1803 y 1810. Más adelante en la época republicana se realizaron seis censos más: 1825, 1835, 1843, 1851, 1864 y 1870 y sucesivamente se han venido realizando muchos más. Históricamente Colombia se ha constituido en uno de los países con mayor tradición de levantamiento de censos de América, al principio estos censos de población eran muy sencillos con categorías simples como sexo y raza. (Secretaria de Planeacion Bogota, 2009)

Para este estudio fue necesario el levantamiento completo de las distintas tipologías existentes en la pieza urbana Sector El Prado del Distrito de Barranquilla, de manera correlacionada con los precios de suelo que actualmente se manejan en el sector de estudio, a fin de establecer una línea base de indicadores urbanos, pertinentes con la visión y acciones de desarrollo del sector y la 
ciudad de Barranquilla. Se presentó la identificación predio a predio junto con las tipologías de viviendas y valores de suelos actuales; todos los datos debidamente geo-referenciados y sistematizados. Lo que se buscaba era identificar la coherencia existente entre los tipos de vivienda y calidad en su diseño de formas y analizar cómo éste, de alguna manera, se ve representado en su valor real referencial, desde las bases normativas que rigen esa dinámica urbana e inmobiliaria. Existen una diversidad de aproximaciones empíricas al desenvolvimiento cuantitativo de los costos del suelo urbano, sobre todo en el estudio de los cambios de esos precios de un mismo terreno a lo largo del tiempo, lo cual es muy importante ya que enseña la evolución del precio del suelo como un activo económico, llegando a configurar las características del mercado inmobiliario y la configuración de la ciudad (Jaramillo, 2014).

Por su parte Barranquilla a finales del siglo XIX e inicios del siglo XX sufrió una serie de cambios y trasformaciones de carácter social y económico, ya que se había establecido como puerto marítimo y fluvial. Esto trae como consecuencia que tancia a Mompox y tome el liderazgo económico reemplazando a Cartagena, que hasta ese momento era la ciudad líder de la costa caribe. Luego en los primeros años del siglo XX, Barranquilla se estableció como la ciudad más importante a nivel económico regional, trayendo esto consigo una importante migración proveniente de otros departamentos del caribe colombiano e incluso del interior del país. (Agudelo y Chapman, 2012)

De esta manera se generó un producto del sector pieza de El Prado con unas aproximaciones a los tipos de predios y sus respectivos valores de suelo actualizados. Los recorridos de manzana a manzana como reconocimiento del sector de estudio, observando sus predios y elaborando registros fotográficos para su análisis fueron una herramienta de gran importancia para llegar a conclusiones relevantes.

\section{Metodología}

La investigación se desarrolló con la siguiente estructura lógica y metodológica: Una primera etapa basada en la observación de campo de reconocimiento y validación de datos planimétricos y datos descriptivos 


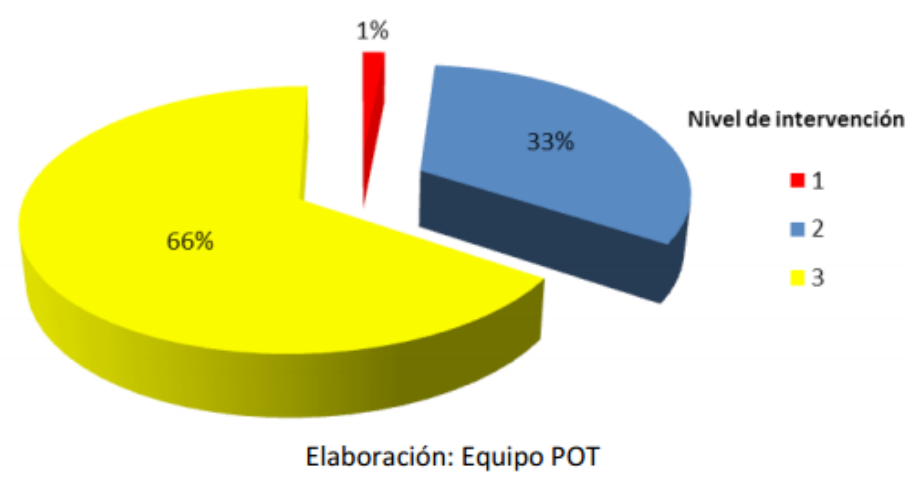

Gráfico 1. Porcentajes de niveles de intervención del Prado

Fuente: POT. Anexo 5: Condiciones urbanísticas para los sectores patrimoniales

de fuentes primarias, seguida de la revisión y recopilación de datos por medio del análisis de datos e indicadores urbanos de usos. Se utilizó también el método del análisis morfológico y tipológico al identificar las características formales del sector en términos de traza, estilos viales, calidad espacial, valor del patrimonio, tipologías y valores. Siguiendo el orden de las actividades planeadas se ha recopilado información existente relacionadas con el indicador de viviendas, en cuanto a morfología urbana y tipología arquitectónica del sector, su historia, a partir de los años 1920, su equipamiento urbano, usos del sector de estudio, entre otros. Al final, se generaron mapas de valores del sector de estudio y sus categorías respectivas.

\section{Análisis de resultados}

Desde el POT Barranquilla (2013) se viene desarrollando las bases para el tratamiento urbanístico de consolidación en zonas con declaratoria patrimonial. Este componente tiene por objeto la recuperación de un sector urbano, mediante obras y modificaciones a los inmuebles que, sin desvirtuar sus condiciones originales, urbanas y arquitectónicas, mejoren sus cualidades funcionales, estéticas, estructurales, de habitabilidad o de confort. La consolidación puede incluir acciones adicionales tales como programas amplios de recuperación o mejoramiento de los espacios públicos y dotación de equipamiento urbano necesario (Secretaria de Planeacion de Barranquilla, 2013). 
Tabla 1

Análisis de valores Manzana 1 El Prado.

\begin{tabular}{|c|c|c|c|c|c|c|c|}
\hline $\begin{array}{l}\text { NUM } \\
\text { MANZĀN }\end{array}$ & $\begin{array}{l}\text { MANZANA } \\
\text { CATASTRO }\end{array}$ & $\begin{array}{c}\text { CODIGO } \\
\text { CATASTRAL } \\
\text { PREDIO }\end{array}$ & $\begin{array}{l}\text { MANZANAS } \\
\text { DIRECCIONE }\end{array}$ & AVALUO & Nivel & $\begin{array}{l}\text { Promedio } \\
\$ / \mathrm{m} 2\end{array}$ & $\begin{array}{c}\text { AREA } \\
\text { M2 }\end{array}$ \\
\hline & & 08001010102530004 & & $\$ 157.611 .000,0$ & 3 & & 319,4 \\
\hline & & 08001010102530002 & & $\$ 202.506 .000,0$ & 3 & & 347,3 \\
\hline & & 08001010102530003 & & $\$ 248.123 .000,0$ & 3 & & 461,8 \\
\hline & & 08001010102530005 & & $\$ 329.614 .000,0$ & 2 & & 658,7 \\
\hline & & 08001010102530006 & & $\$ 315.442 .000,0$ & 2 & & 859,6 \\
\hline & & 08001010102530008 & & $\$ 323.372 .000,0$ & 2 & & 707,2 \\
\hline & & 08001010102530009 & & $\$ 143.170 .000,0$ & 2 & & 415,6 \\
\hline \multirow[t]{2}{*}{1} & 0800101010253 & 08001010102530007 & $\begin{array}{l}\text { K. 54-k.53- } \\
\text { C.53.C54 }\end{array}$ & $\$ 242.257 .000,0$ & 3 & 394208,2 & 647,6 \\
\hline & & 08001010102530012 & & $\$ 245.731 .000,0$ & 2 & & 763,6 \\
\hline AREA M2 & & 08001010102530010 & & $\$ 305.326 .000,0$ & 2 & & 1084,0 \\
\hline \multirow[t]{4}{*}{8933} & & 08001010102530011 & & $\$ 168.108 .000,0$ & 2 & & 461,7 \\
\hline & & 08001010102530013 & & $\$ 279.601 .000,0$ & 2 & & 753,1 \\
\hline & & 08001010102530901 & & $\$ 560.601 .000,0$ & 3 & & 1453,5 \\
\hline & & & & $\$ 3.521 .462 .000,0$ & & TOTAL & 8933,0 \\
\hline
\end{tabular}

Fuente: Elaborado por el autor (2014).

En el sector del Prado, Alto Prado y Bellavista se encontraron bienes patrimoniales compuestos principalmente por estatuas y monumentos, los cuales se ubican sobre las vías principales y parques representativos del sector, con lo que se complementa el sistema de espacio público, al establecer ciertos puntos de encuentro y referencia para la ciudad y por ende resaltan el valor urbanístico de estas zonas (Secretaria de Planeacion de Barranquilla, 2013).
En dicho documento se definieron puntualmente los tipos de intervención a los que está sujeto todo trabajo de análisis e intervención urbanística del sector de estudio. A saber:

Nivel 1. Conservación integral: Se aplica a inmuebles de excepcional valor, los cuales, por ser irremplazables, deben ser preservados en su integralidad. En estos, cualquier intervención puede poner en riesgo sus valores e integridad, por lo que las obras deben ser 


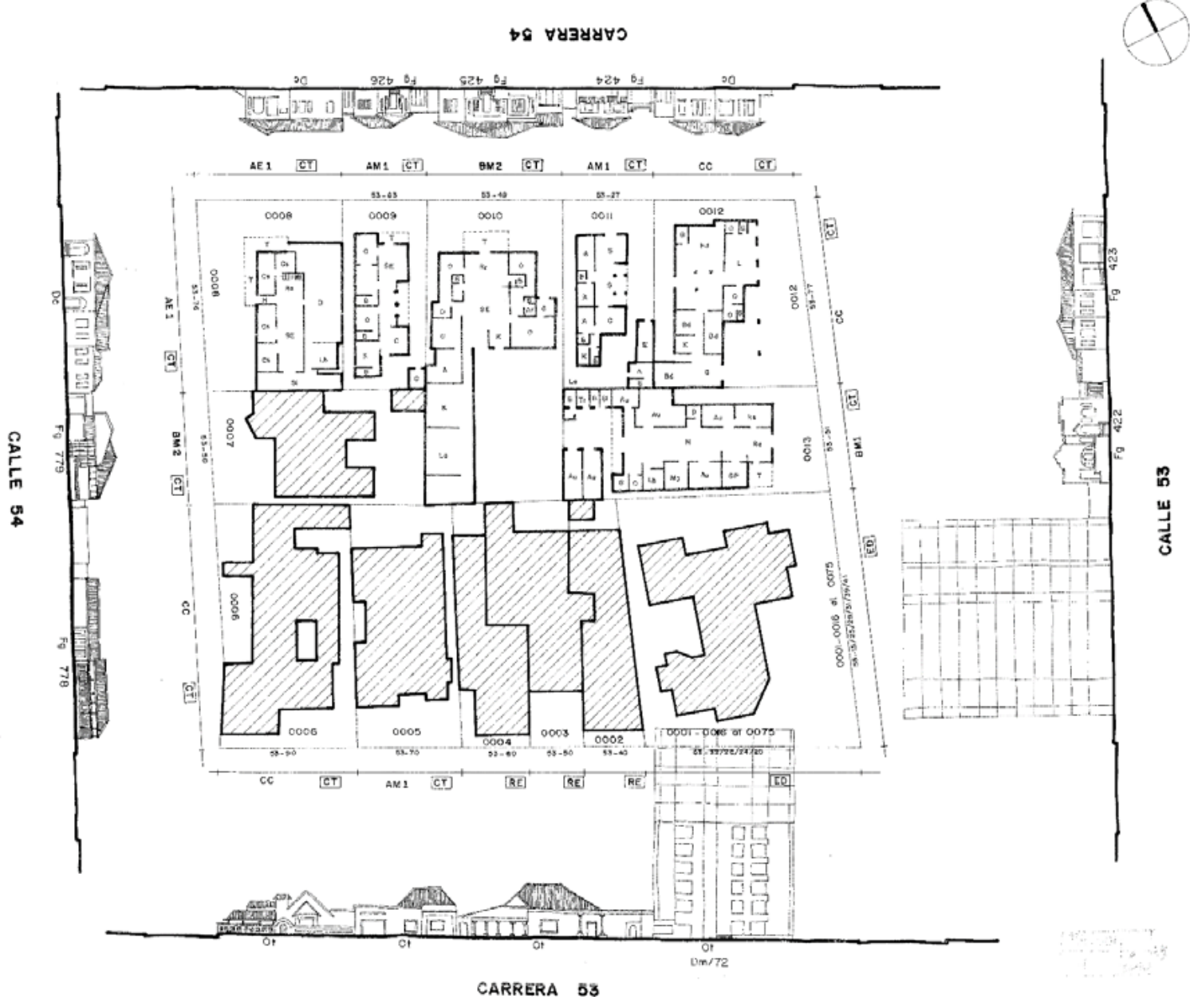

Figura 1. Imagen de plano catastral manzana 1 sector El Prado.

Fuente: IGAC (2014)

legibles y dar fe del momento en el que se realizaron.

Nivel 2. Conservación del tipo arquitectónico: Se aplica a inmuebles con características representativas en términos de implantación predial (rural o urbana), volumen edificado, organización espacial y elementos ornamentales las cuales deben ser conservadas. Se 


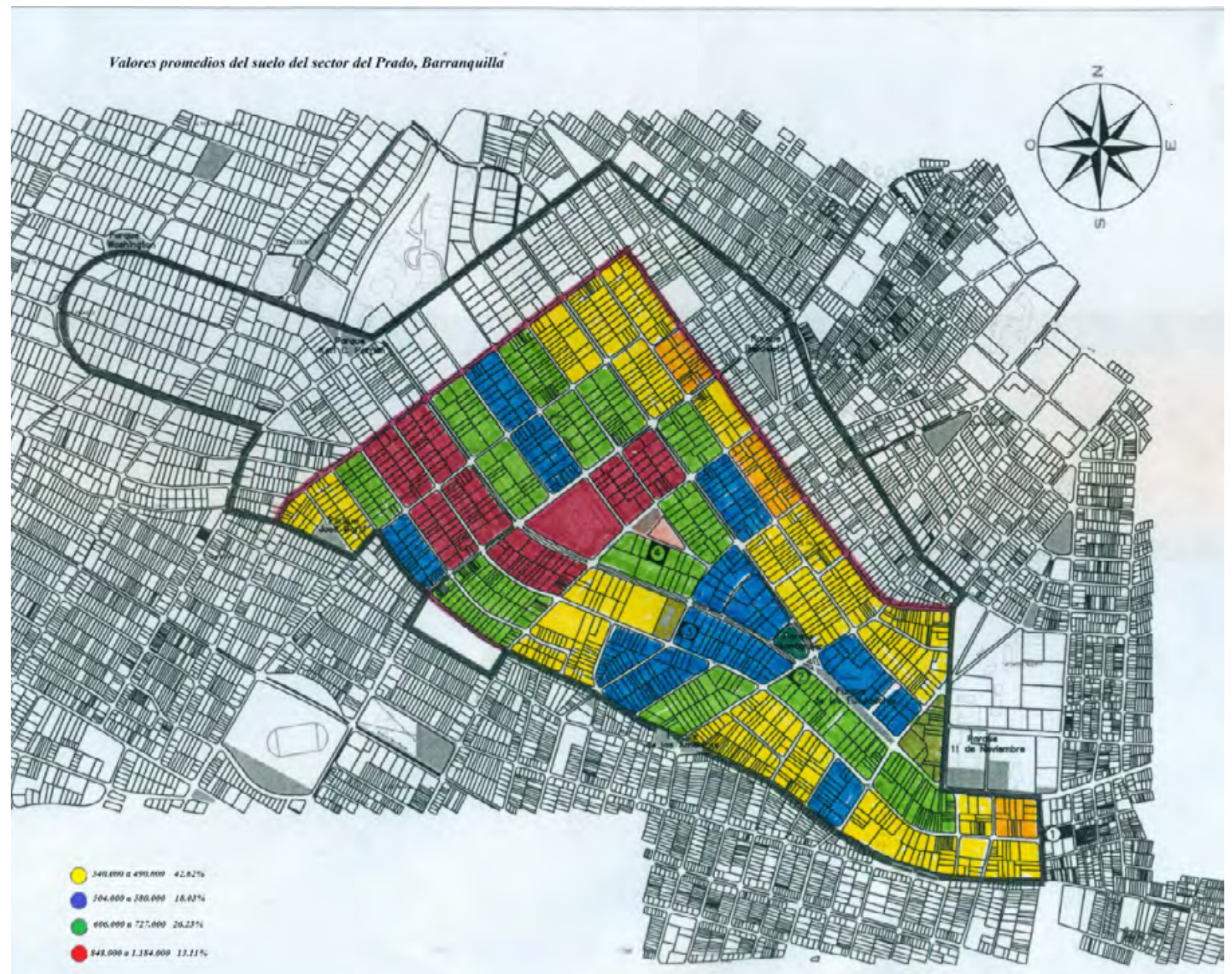

Figura 2. Imagen plano de valores promedio del suelo urbano del sector El Prado

Fuente: Elaborado por el autor (2014).

de su estructura espacial: disposición de accesos, vestíbulos, circulaciones

horizontales y verticales. permite la modificación de los espacios internos del inmueble, siempre y cuando se mantenga la autenticidad 


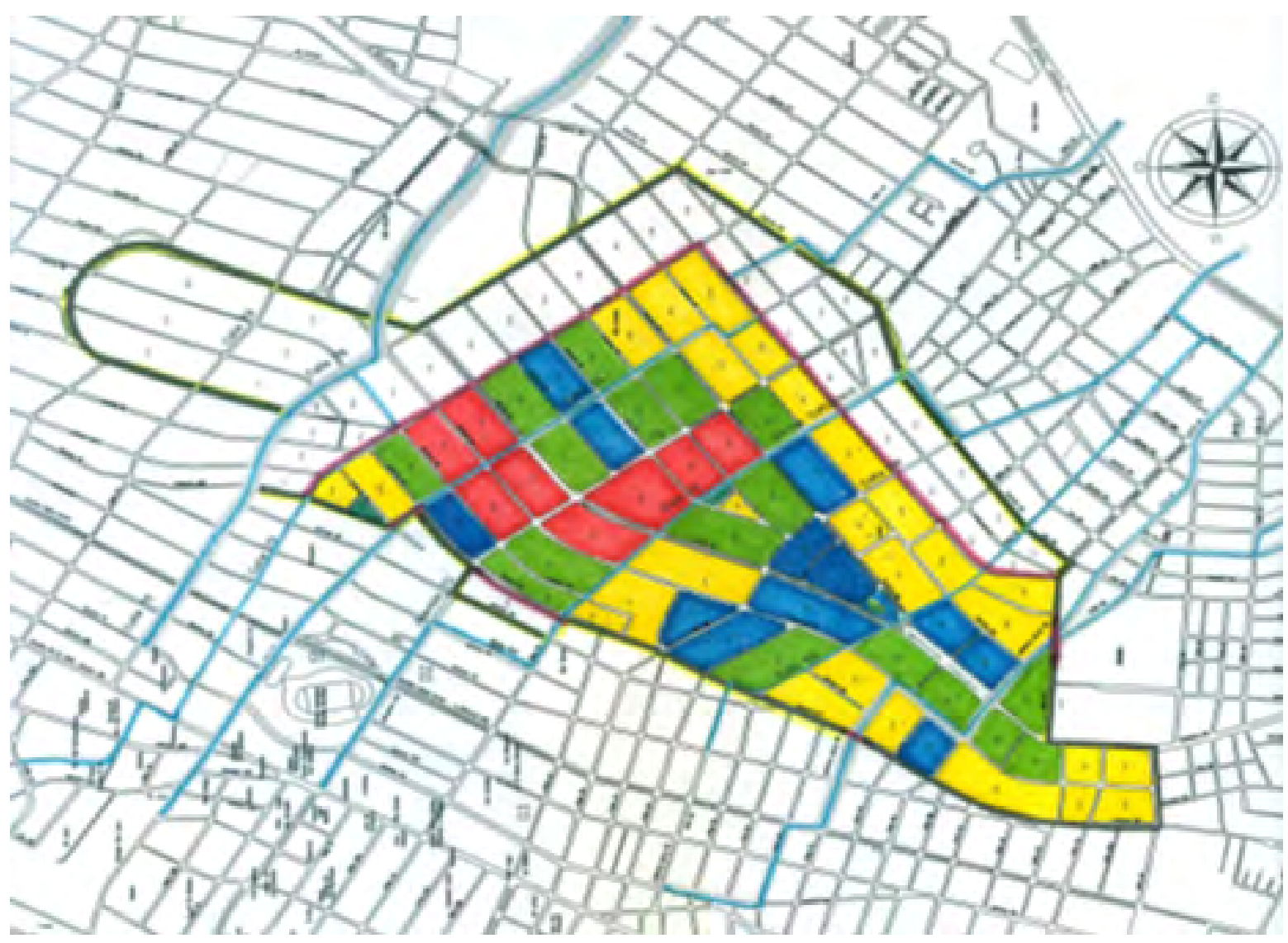

Figura 3. Valores Promedios del Suelo de las Manzanas del Sector del Prado Fuente: Elaborado por el autor (2014).

Nivel 3. Conservación contextual: Se aplica a inmuebles ubicados en un Sector Urbano, los cuales, aun cuando no tengan características arquitectónicas representativas, por su implantación, volumen, perfil y materiales, son compatibles con el contexto. De igual manera, se aplica para inmuebles que no son compatibles con el contexto, así como a predios sin construir que deben adecuarse a las características del sector urbano. Este nivel busca la recuperación del contexto urbano en términos del trazado, perfiles, paramentos, índices de ocupación y volumen edificado. 
La investigación se basó en el uso de tablas de análisis y datos numéricos para establecer aproximaciones en cuanto a los usos y valores del suelo.

Con base en los modelos de tablas anteriores e pudo identificar un promedio de valor que ayuda a identificar por manzanas los valores prediales y su nivel de intervención. En el caso de la estadística de valores en la manzana 1, así como las 60 restantes que comprenden la pieza urbana El Prado, se analizó la información catastral por cada predio del sector, tal como se muestra a continuación:

Los datos catastrales permitieron identificar y cuantificar los índices de valor por manzana, tomando como partida las cantidades, estado, distribución y categorías de intervención patrimonial de cada unidad por manzana.

A partir de los datos anteriores, la investigación pudo generar los promedios generales que dan con una descripción global pero específica de cada manzana y predio en el contexto urbano de El Prado.

Las convenciones generadas para dichos se describen a continuación,
Tabla 2

Análisis de valores promedio categorizados EI Prado.

\begin{tabular}{ll}
\hline $340.000-490.000$ & $42.62 \%$ \\
\hline $504.000-580.000$ & $18.03 \%$ \\
\hline $848.000-1.184 .000$ & $13.11 \%$ \\
\hline $606.000-727.000$ & $26.23 \%$ \\
\hline
\end{tabular}

Fuente: Elaborado por el autor (2014).

El Prado presenta agrupaciones categorizadas por valores del suelo, que se desarrollaron según los precios y actividades propias de la economía local.

Se pudo predefinir a nivel de prospectiva económica del sector El Prado que hay un crecimiento en los rangos de valor entre $340.000-1.184 .000$ millones de pesos, asociados con actividades comerciales, institucionales, educativas y habitacionales. Con respecto al margen legal que define toda actividad de intervención de la pieza urbana, el POT plantea una clara perspectiva de conservación y rescate de los valores patrimoniales de El Prado, que en cierta manera coaccionan la dinámica económica y social del sector en el contexto de desarrollo local y nacional. 


\section{Conclusiones}

Se consideró que toda acción de valoración económica de inmuebles con valor patrimonial constituye una herramienta de análisis muy útil para el gobierno local y para el campo de la gestión del patrimonio, teniendo en cuenta que el desarrollo urbano actual en Barranquilla propone la reactivación, renovación e incluso, un modelo de desarrollo para una ciudad sostenible, encargada de equilibrar la potenciación de todos los territorios según sus propias aptitudes.

La investigación urbana propicia la apertura de nuevas líneas base oportunas para el trabajo de valorización y desarrollo urbano de la pieza urbana El Prado al proporcionar elementos de juicio veraces y reales que puedan soportar la toma de decisiones y los modelos de desarrollo o intervención urbanística del Prado, partiendo de sus elementos actuales y estado de conformación física y económica, con relación a las prospectivas de visión de la ciudad de Barranquilla. En todo caso los valores del suelo en los barrios del norte de Barranquilla presentan una valorización superior reflejo de la dinámica de inversiones en los últimos años debido a la demanda de suelo para construcción de vivienda y comercio y esto ha permitido un crecimiento estable y natural. Por el contrario, los barrios del sur occidente y sur oriente presentan valorizaciones inferiores a las esperadas. (Payares, 2012)

A través de la realización estudio de valores del suelo del Prado se pudo establecer la validación del modelo y procedimiento aplicado en la valoración desde el punto de vista económico de las construcciones patrimoniales, por medio de reconocimiento de las edificaciones, identificación de los niveles de intervención, avalúos inmobiliarios, y cuantificación de áreas de manzanas y predios; esto, permitió arrojar las aproximaciones de valores que establecen un mapeo de las zonas y la distribución de los valores globales por manzanas.

Los datos permitieron afirmar que El Prado presenta valorizaciones globales distribuidas en 4 rangos a nivel de predio-manzana. Los de mayor valor se encuentran en rangos de 800.000 a 1.200.000 millones; en segundo rango están en 600.000 a 800.000; tercer rango de 500.000 a 600.000; y el último rango entre 300.000 a 500.000 . 
Los valores establecidos parten del análisis y cálculo del Valor del suelo, del Valor catastral y su ponderación con del número de predios por manzana, permitieron distribuir por convenciones los rangos de precios y su concentración dentro del sector, a partir de un mapa de valores y comportamientos como producto final de la investigación.

\section{Referencias}

Agudelo, A. y Chapman, W. (2012). Que el señor alcalde haga destruir las casas pajizas: el proceso de transformacion urbana en Barranquilla a finales del siglo XIX y principios del siglo XX. Memorias, 197-223.

Alfonso, D. y Erba, M. (2015). Catastrolatino.org. Recuperado de http://www.catastrolatino.org/ documentos/bogotanov2005/ ponencia_Instituto_Linconl.pdf
Jaramillo, S. (2014). Dinámica de los precios del suelo urbano. CEDE, 6-58.

Ospino, P. (2003). El desarrollo urbano de Barranquilla y su dinamica regional 1777-1993. En L. Sanchez, Barranquilla: Lecturas urbanas (pp. 3-43). Bogota: Observatorio del Caribe colombiano- Uiversidad del Atlantico.

Payares, D. (2012). Estimacion del potencial de valorizacion del suelo en Barranquilla en el periodo 20012011. Revista de economia del caribe, 65-91.

Secretaria de Planeacion de Bogotá. (2009). Conociendo Bogota y sus localidades. Diagnostico de los apsectos fisicos, demograficos $y$ socioeconomicos. Bogota: Alcaldia Mayor de Bogota.

Secretaria de Planeacion de Barranquilla. (2013). Plan de ordenamiento territorial de Barranquilla. Barranquilla: Alcaldía de Barranquilla. 